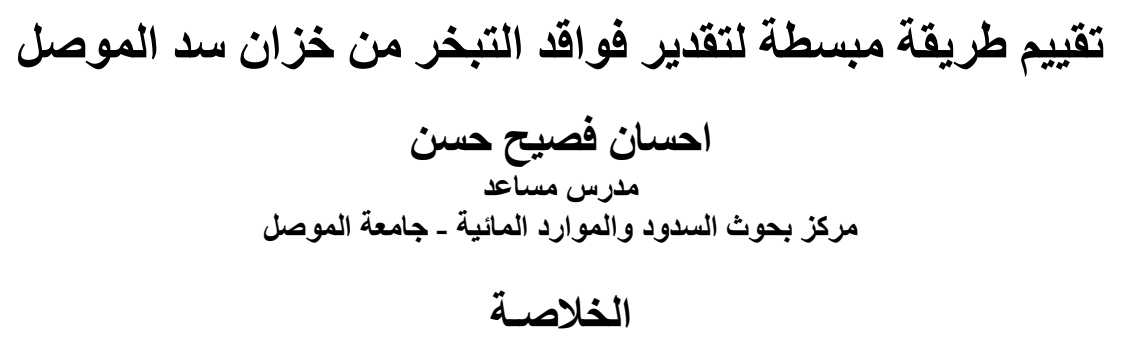

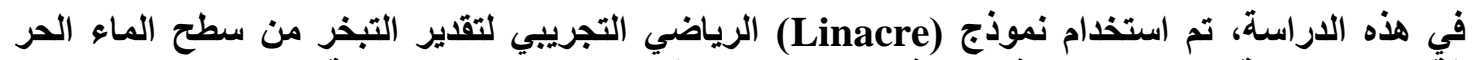

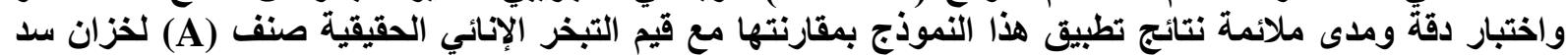

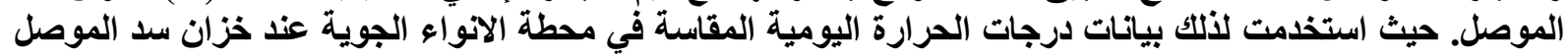

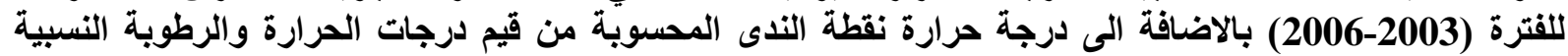

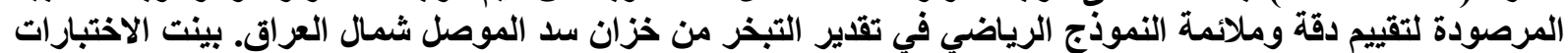

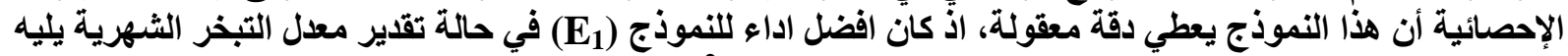

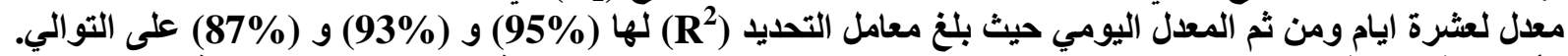

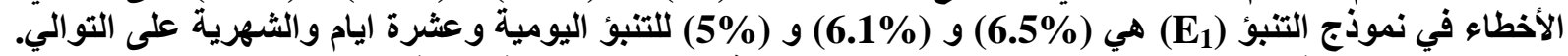

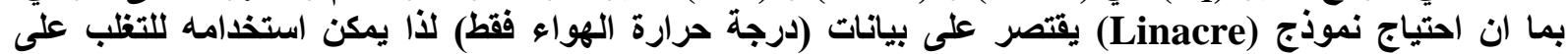
صعوبة النقص في البيانات المتاحة في محطات الأنواء الجوية.

\title{
Evaluation of a Simplified Method to Estimate Evaporation Losses From Mosul Dam Reservoir
}

\author{
Mr. Ihsan F. Hasan \\ Assistant Lecturer \\ Dams and Water Resources Research Center/University of Mosul
}

\begin{abstract}
In this study, the (Linacre) mathematical Pilot model was used to estimate evaporation from free water surface and test the results accuracy of the mathematical evaporation model by comparing it with the values of the measured pan evaporation class (A) at the Mosul dam reservoir. The daily measured temperature data recorded in meteorological station at the Mosul dam reservoir for the period (2003-2006) were used in addition to the calculated dew point temperature from the values of measured temperature and relative humidity to verify the accuracy of the mathematical model in estimating the evaporation from the Mosul dam reservoir northern of Iraq. Statistical tests showed that this model gives a reasonable results. The accuracy of the model is best for the monthly rate evaporation prediction (E1), followed by the rate of ten days and then daily, with coefficient of determination $\left(R^{2}\right)$ are $(95 \%)$ and $(93 \%)$ and $(87 \%)$, respectively. The percent errors in the model prediction (E1) are $(6.5 \%)$ and $(6.1 \%)$ and (5\%) for daily, ten days and monthly prediction respectively. Since the (Linacre) model require very few data (air temperature only), it can be used to overcome the difficulty of lack of the meteorological data.
\end{abstract}

Key word: Linacre model, Mosul dam reservoir, Pan Evaporation. 


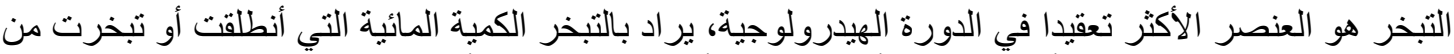

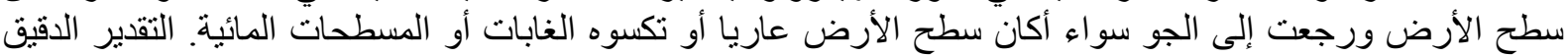

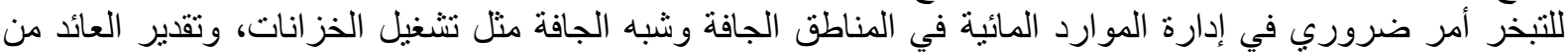

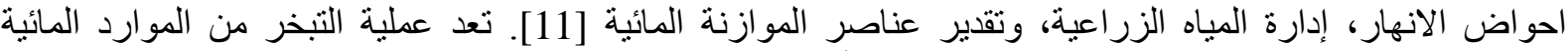

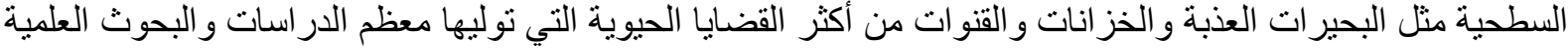

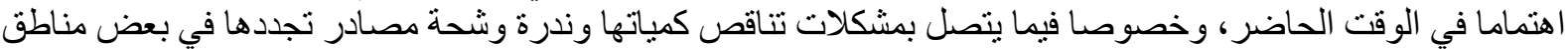

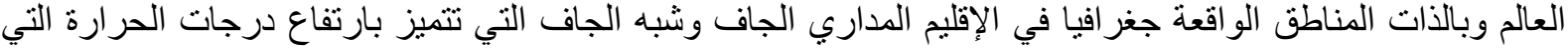

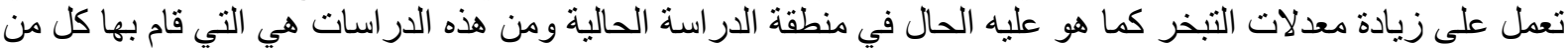

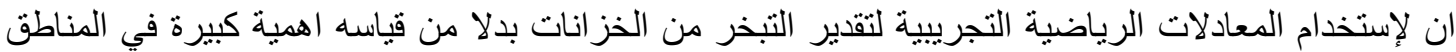

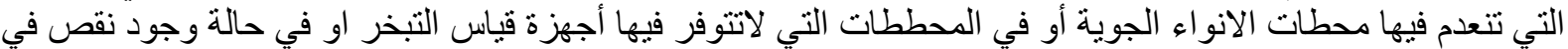

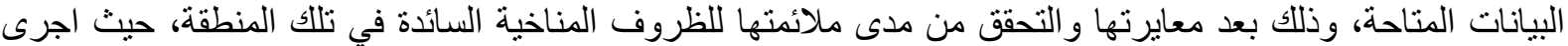

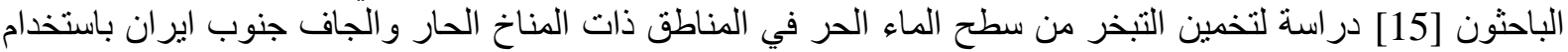

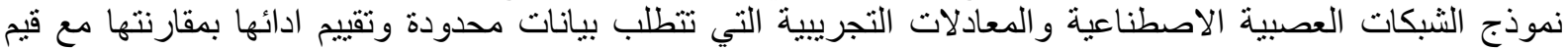

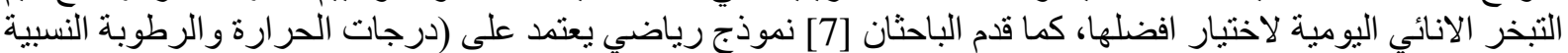

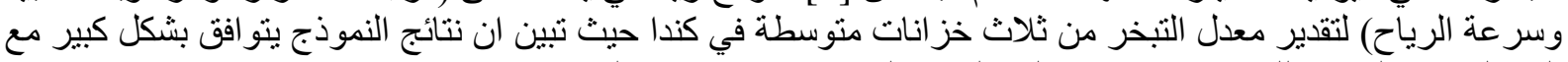

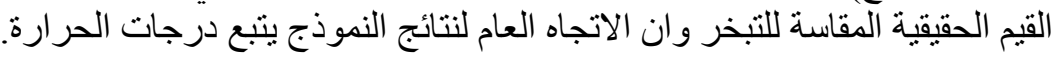

\section{منطقة الاراسة والبيانات المناخية}

يمثل موقع الدراسة خزان سد الموصل الو اقع على نهر دجلة في الجزء الثمالي من العر اق اق على بلى بعد حو الي (60)

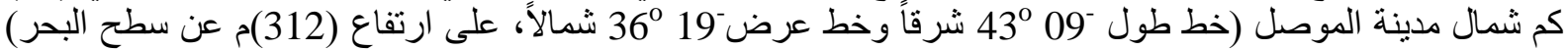

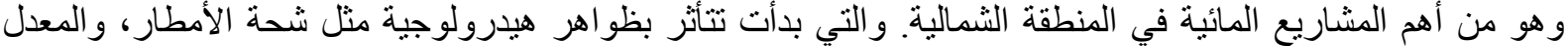

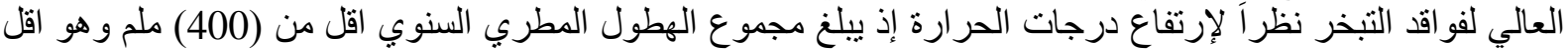

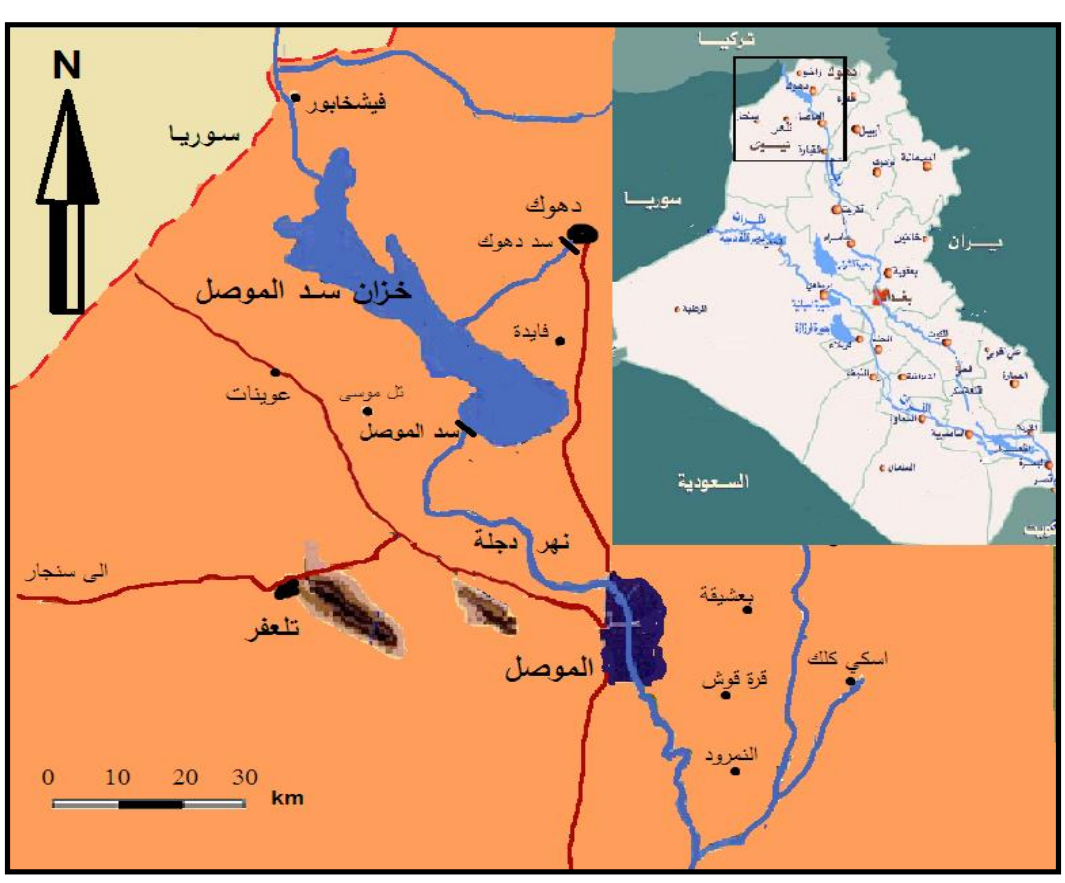

شكل (1) يبين موقع خزان سد الموصل

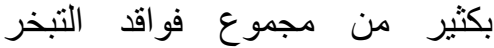
السنوي البالغ حوالي (2000) ملم من النيخ

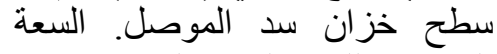
التخزينية للسد تبلغ حوان الي (11100)

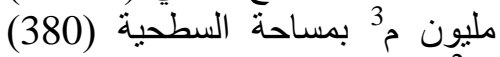

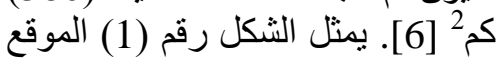
العام لمنطقة الدر اسة:

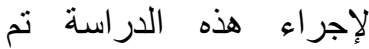
اعنماد البيانات المناخية المسجلة من الأنة

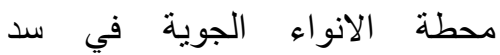

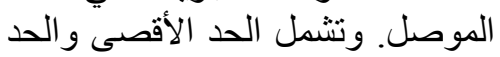

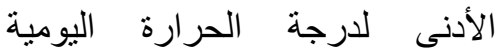
والرطوبة النسبية وقيم التبخر الإنائي صنف (A) لمدة اربعة سنوات من الآن 2003 إلى 2006. ويبين الثكل (2) الثنان

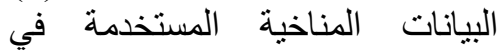

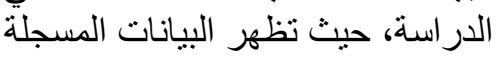
نفس الإتجاه العام لقيم التبخر الإنائي

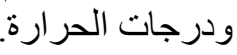


حسن : تقييم طريقة مبسطة لتقدير فواقد التبخر من خزان سد الموصل

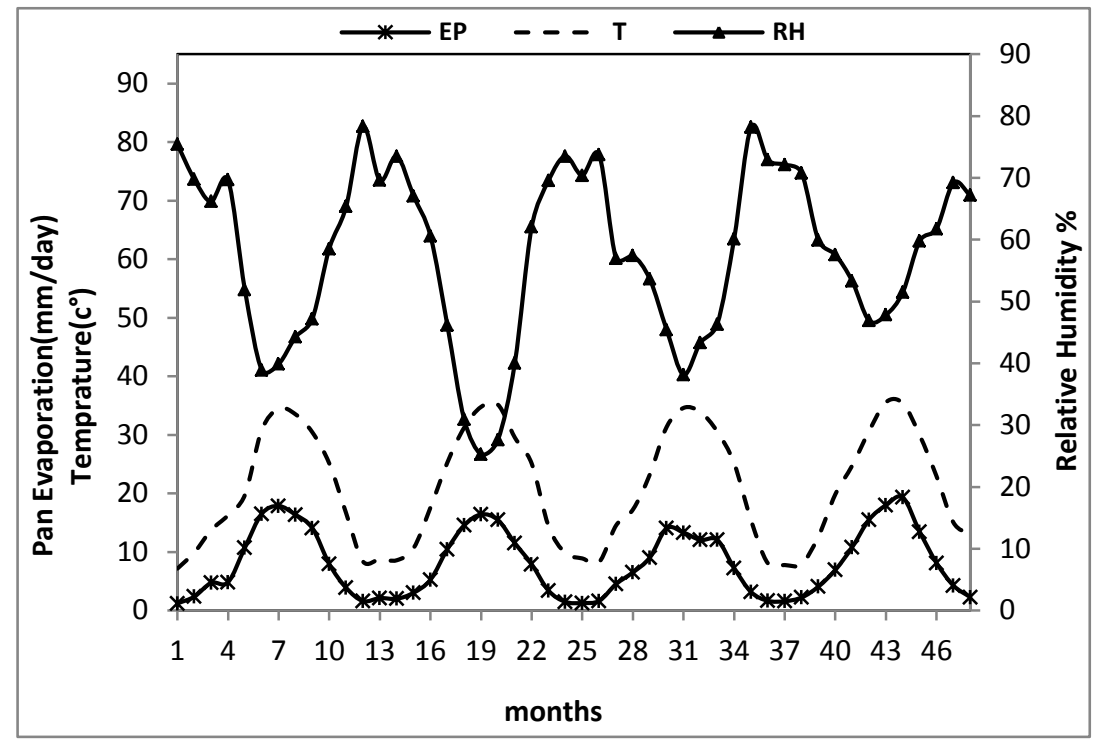

شكل (2) البيانات المناخية المستخدمة في البحث في موقع سد الموصل

تطبيق نموذج (Linacre)

هنالك نماذج كثيرة لتقدير التبخر من سطح المياه المفتوحة، والتي تتضمن تقدير التبخر من البحيرات الطبيعية

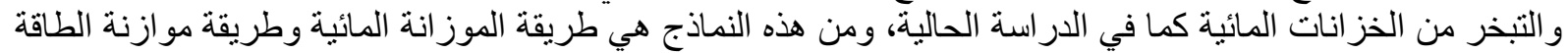

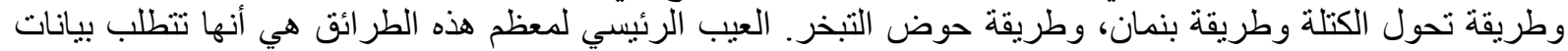

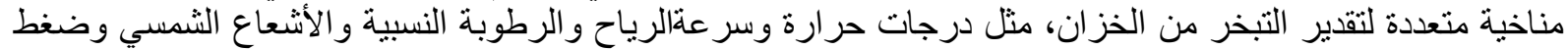

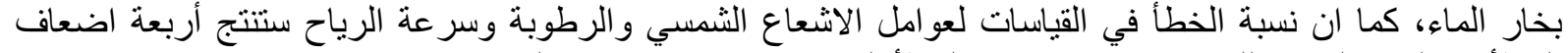

الخطأ في القيمة المقدرة للتبخر مقارنة مع نفس الخطأ الناتج من قياسات درجات الأن الحرارة [2].

وفي محاولة للتغلب على الصعوبة المر افقة في استخدام معادلة بنمان التي تتطلب بيانات مناخية متعددة، قدم

Linacre

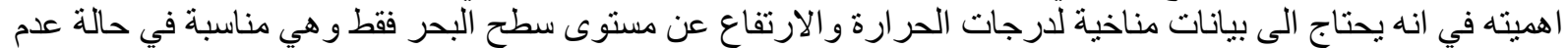
توفر بيانات مناخية متعددة [13]. ومعادلة بنمان للتبخر هي:

$\mathrm{LE}=\left(\mathrm{R}_{\mathrm{n}}+\rho \mathrm{C}\left(\mathrm{e}_{\mathrm{s}}-\mathrm{e}_{\mathrm{a}}\right) / \Delta \mathrm{r}_{\mathrm{a}}\right) /(1+\gamma / \Delta)$

$$
\text { LE }
$$

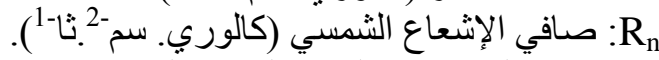
e e

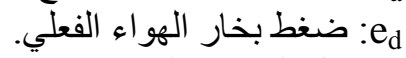
د: ميل المنحنى المرسوم بين ضغاء الفيط البخار عند التشبع ودرجة الحرارة ra

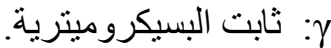

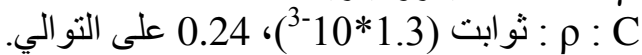

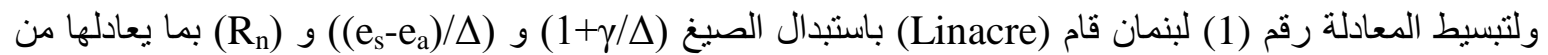

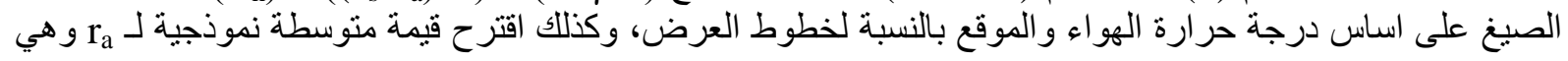


$(1+\gamma / \Delta)=2(1-0.0125 \mathrm{~T})$

$\left(\mathrm{e}_{\mathrm{s}}-\mathrm{e}_{\mathrm{a}}\right) / \Delta=\left(\mathrm{T}-\mathrm{T}_{\mathrm{d}}\right)$

$\left.\mathrm{R}_{\mathrm{n}}=0.55(\mathrm{~T}+\mathrm{O} .006 \mathrm{~h}) / 60(100-\mathrm{A})\right)$

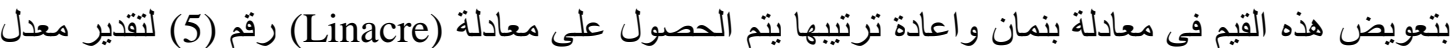

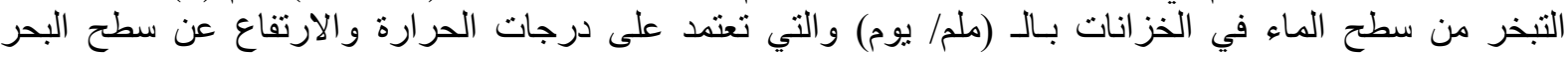
و الاحداثيات الجغر افية للمنطقة فقط. [13

$E=\frac{700 *(T+0.006 h) /(100-A)+15\left(T-T_{d}\right)}{80-T}$

$$
\text { قدم (Linacre) في در استه معادلة أخرى التقدير (T - Td ) على النحو الاتي: }
$$

$\left(T-T_{d}\right)=0.0023 h+0.37 T+0.53 R+0.35 R_{\text {ann }}-10.9$

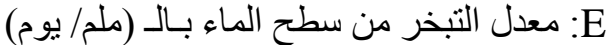

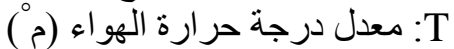

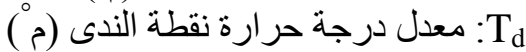

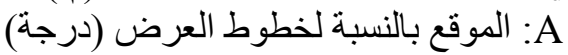

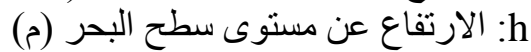

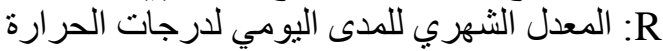
R: Rann

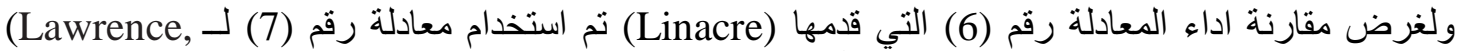

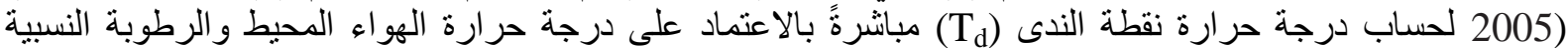

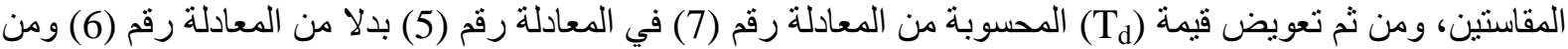
$T_{d}=\frac{b[\ln (R H / 100)+a * T /(b+T)]}{a-\ln (R H / 100)-a * T /(b+T)}$

$0 \mathrm{C}^{\circ}<\mathrm{T}<60 \mathrm{C}^{\circ}$

$1 \%<\mathrm{RH}<100 \%$

$0 \mathrm{C}^{\circ}<\mathrm{T}_{\mathrm{d}}<50 \mathrm{C}^{\circ}$ ثم مقارنة قيم التبخر الناتجة في الحالتين.

يعتبر إناء التبخر الأمريكي الأسطواني الثكل صنف (A) هو الاكثر انتشاراً واستعمالا لاى أغلب المحطات

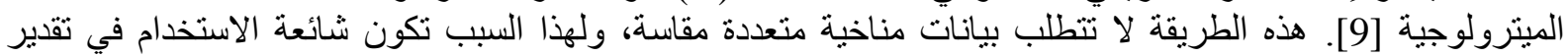
التبخر من خز انات و البحير الطري في المناطق التي تكون فيها البيانات المناخية محدودة او غير متوفرة.

ان تسرب الحرارة من خلال جدار الوعاء واستعمال شبكة من أسلاك التغطية والمادة التي يصنع منها الإناء وكذلك وجود او عدم وجود الغطاء النباتي في المكان الذي يوضع فيه إناء التبخر كلها عو امل يمكن ان تؤثر في كمية الفاقد التاء 
المقاسة من الماء. وعليه لتقدير التبخر من سطح الماء في الخزان نقوم بضرب مقدار التبخر من الإناء بمعامل مناسب

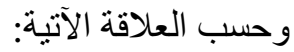

$\mathrm{Ep}=\mathrm{K}_{\mathrm{P}} * \mathrm{E}_{\mathrm{Pan}}$

حيث:

Ep K KP EPan

في هذه الدراسة تم استخدام قيم التبخر المقاسة عند محطة القياس بالقرب من خزان سد الموصل باستخدام إناء

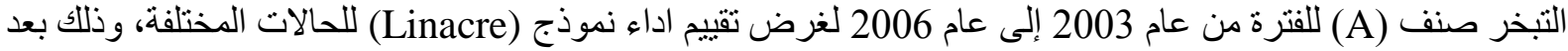

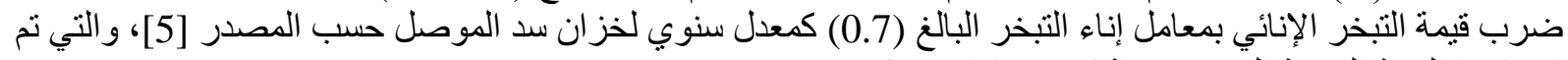
اعتبار ها القيمة الحقيقة المرصودة الثناء تقييم اداء نموذج (Linacre).

\section{(ختبار دقة أداء نموذج (Linacre)}

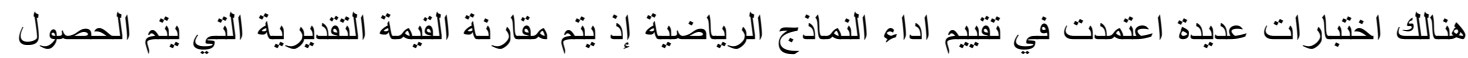

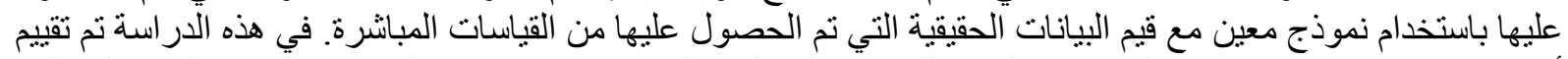

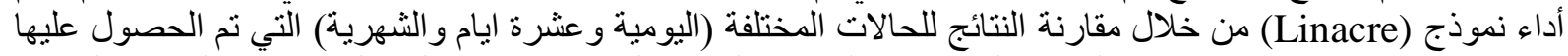

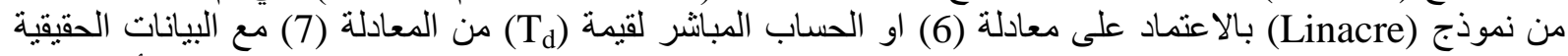

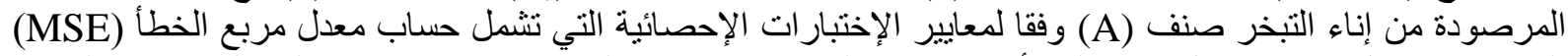

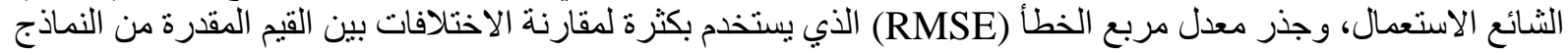

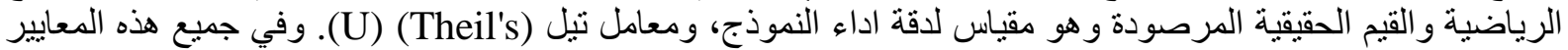

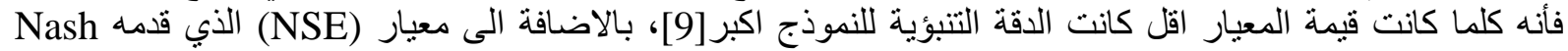

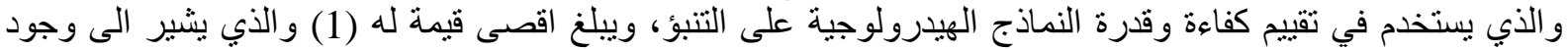

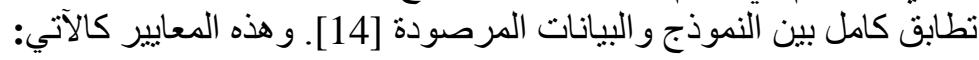

$M S E=\frac{\sum_{i=1}^{N}\left(E i_{\text {obs }}-E i_{\text {pred }}\right)^{2}}{N}$

$$
R M S E=\sqrt{\frac{\sum_{i=1}^{N}\left(E i_{\text {obs }}-E i_{\text {pred }}\right)^{2}}{N}}
$$

$U=\frac{\sqrt{\frac{1}{N} \sum_{i=1}^{N}\left(E i_{o b s}-E i_{\text {pred }}\right)^{2}}}{\sqrt{\frac{1}{N} \sum_{i=1}^{N}\left(E i_{o b s}\right)^{2}}+\sqrt{\frac{1}{N} \sum_{i=1}^{N}\left(E i_{\text {pred }}\right)^{2}}}$

$N S E=1-\frac{\sum_{i=1}^{N}\left(E i_{o b s}-E i_{p r e d}\right)^{2}}{\sum_{i=1}^{N}\left(E i_{o b s}-\overline{E l_{o b s}}\right)^{2}}$

حيث ان

(mm/day) (التبخر الإنائي (Ei

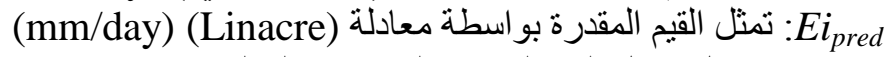

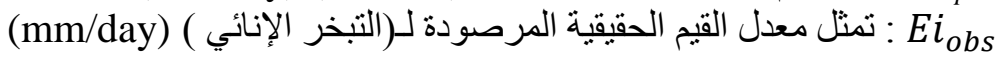


جدول رقم (1) قيم نتائج التحليل الاحصائي للنموذج

\begin{tabular}{|c|c|c|c|c|c|c|c|c|}
\hline \multicolumn{4}{|c|}{$\mathbf{E}_{\mathbf{P}} \& \mathbf{E}_{2}^{*}$} & \multicolumn{4}{|c|}{$\mathbf{E}_{\mathbf{P}} \& \mathbf{E}_{1}^{*}$} & \multirow{2}{*}{ الفترة } \\
\hline NSE & $\overline{\mathbf{U}}$ & RMSE & MSE & NSE & $\overline{\mathbf{U}}$ & RMSE & MSE & \\
\hline $84 \%$ & 0.13 & 2.6 & 6.77 & $80 \%$ & 0.11 & 2.39 & 5.74 & يومي \\
\hline $85 \%$ & 0.12 & 2.17 & 4.72 & $89 \%$ & 0.10 & 1.88 & 3.55 & عشرة ايام \\
\hline $86 \%$ & 0.11 & 2.12 & 4.51 & $90 \%$ & 0.09 & 1.77 & 3.16 & شهري \\
\hline
\end{tabular}

النتائج و المناقثة

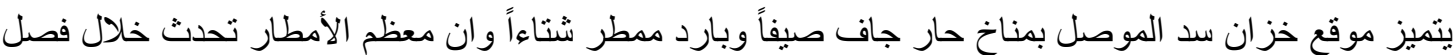
الثتاء. لذا أعلى قيمة للمعدل الثهري للتبخر الإنائي (Ep) تبلغ (19.3) ملم في آب، 2006، 2006، في حين أن أدنى قيمة للمعدل الثهري للتبخر الإنائي (Ep) تبلغ (1.2) ملم في كانون الثناني، 2003.

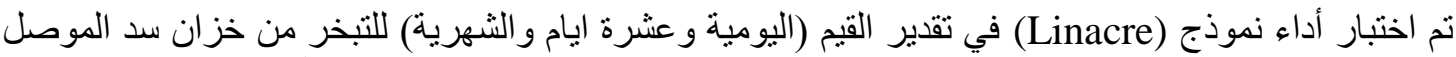

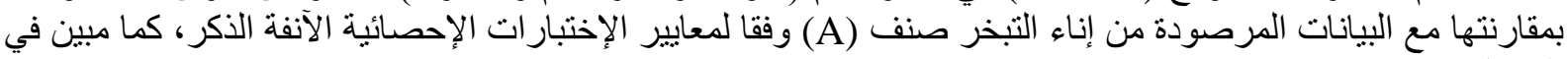

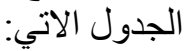

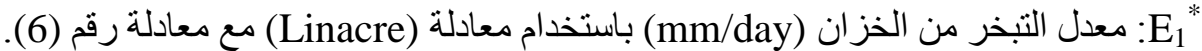

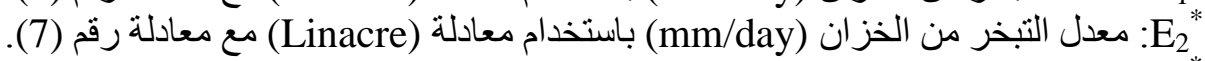

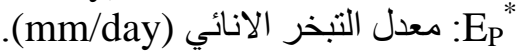

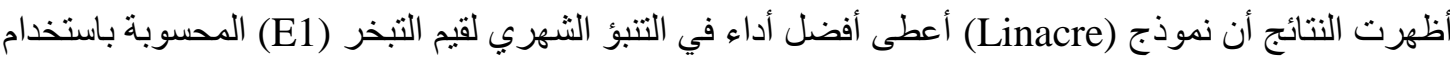

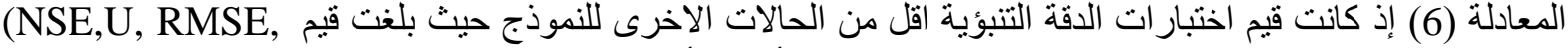
(90\%, 0.09, 1.77, 3.16) MSE)

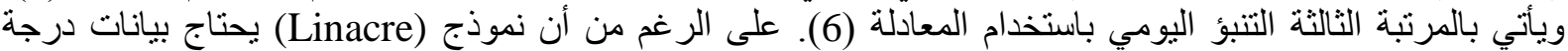

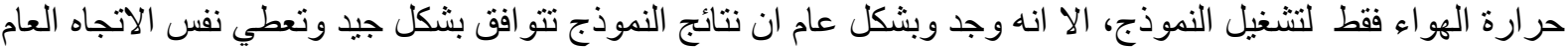

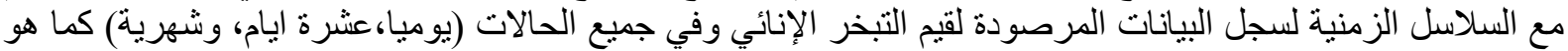

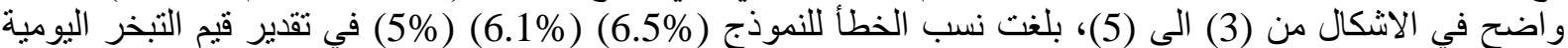

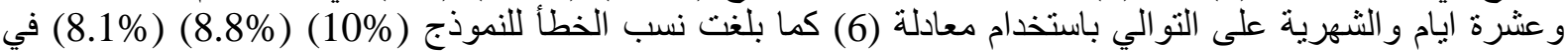
تقدير قيم التبخر اليومية وعشرة اليام و الثهرية على التو الئ باستخدام معادلة (7).

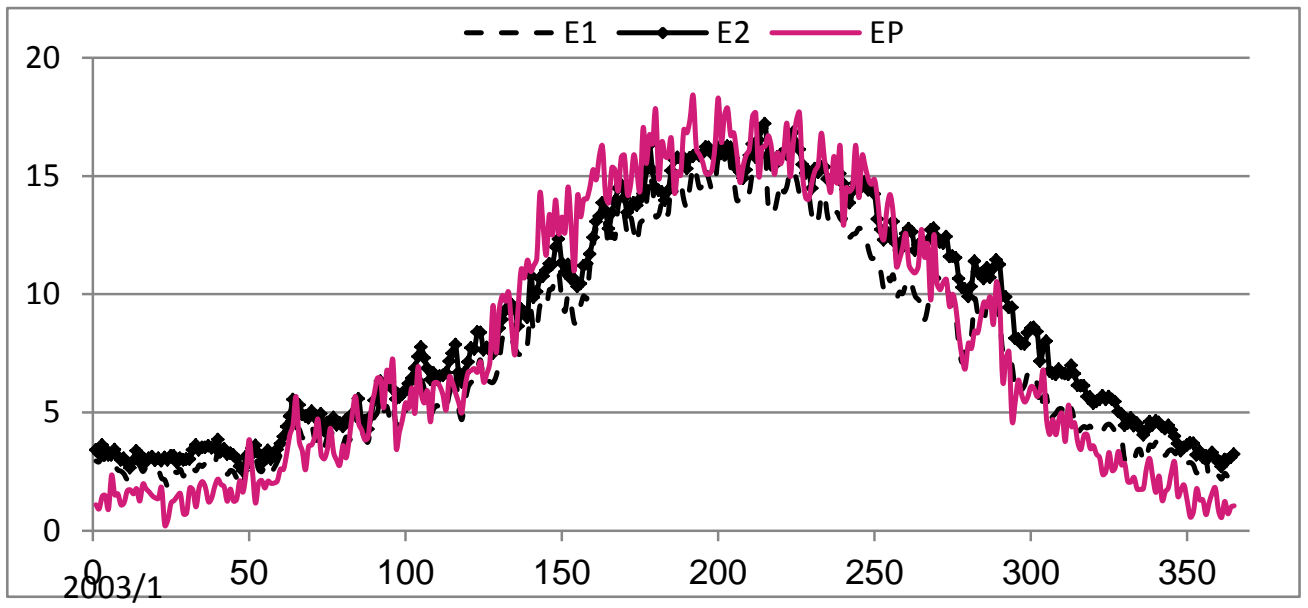

شكل (3) قيم معدل التبخر اليومي الحقيقية و المقدرة باستخدام نموذج (Linacre) لخزان سد الموصل 
حسن : تقييم طريقة مبسطة لتقدير فواقد التبخر من خزان سد الموصل

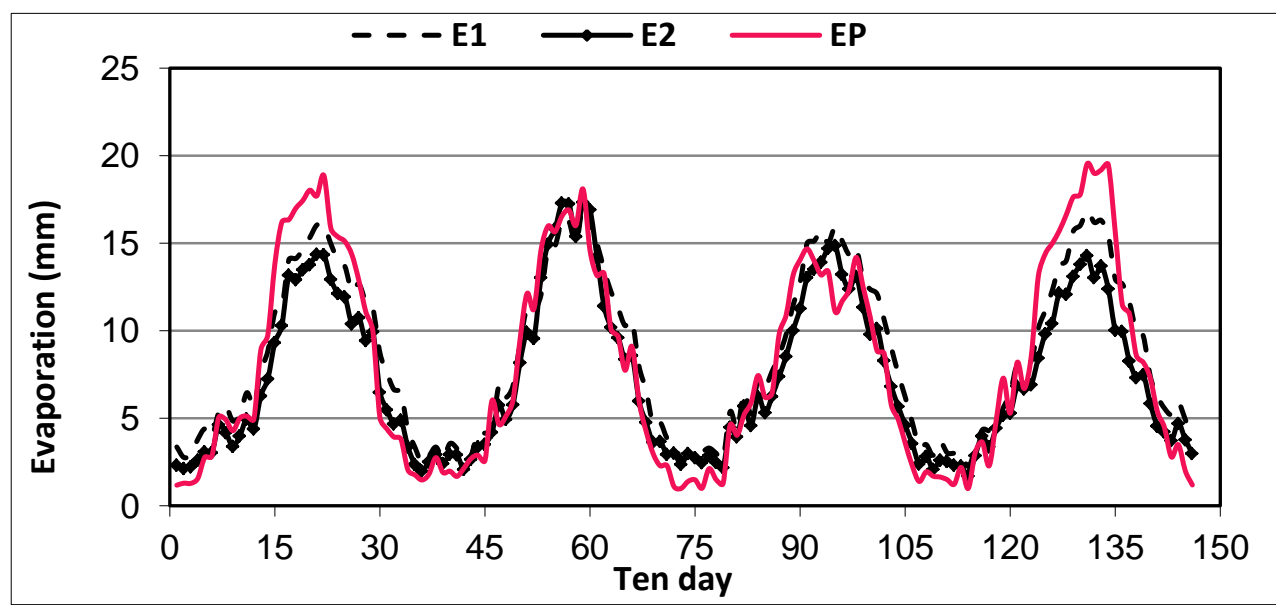

شكل (4) قيم التبخر الحقيقية و المقدرة باستخدام نموذج (Linacre) لعشرة ايام لخز ان سد الموصل

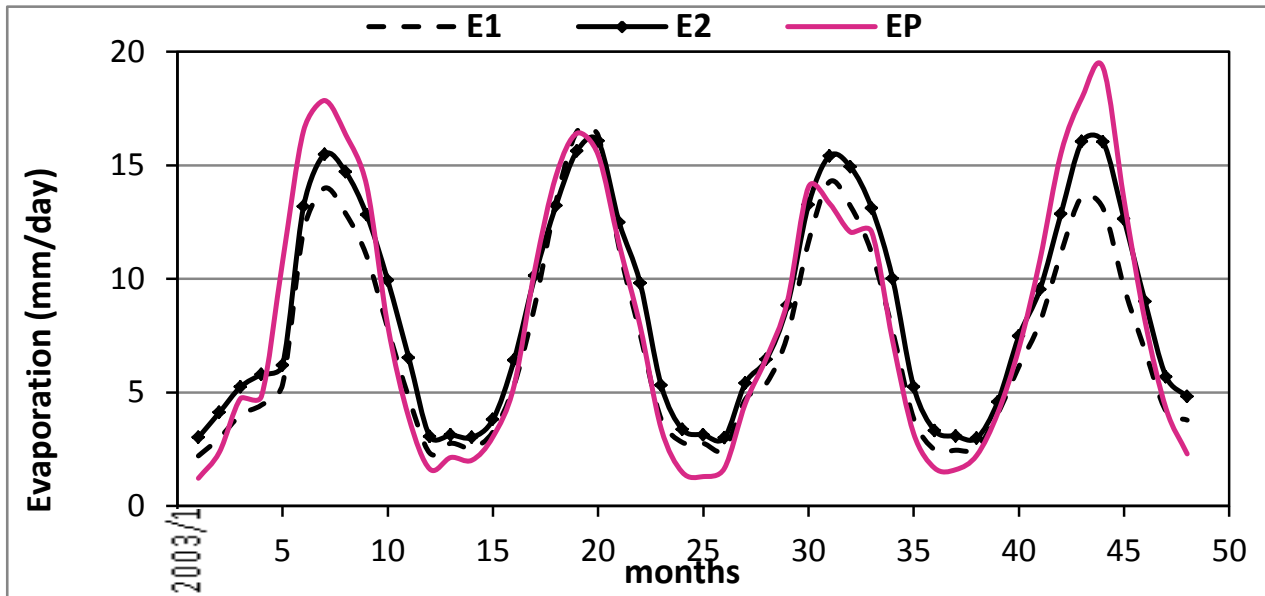

شكل (5) قيم التبخر الحقيقية و المقدرة باستخدام نموذج (Linacre) الثهرية لخزان سد الموصل

للتحقق من صلاحية النموذج لتقدير التبخر من الخزان تم رسم مخططات نقاط التبعثر وايجاد قيم معامل التحديد

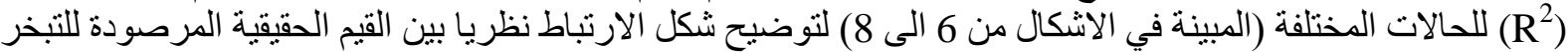

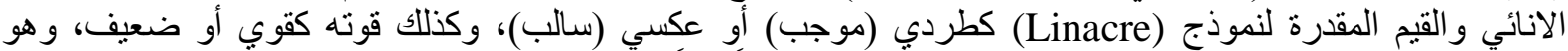
مقياس جيد لفحص التر ابط الخطي للقيم حيث يكون التنر ابط موجباً قوياً إذا كانت نقاط التبعثر (الناتجة من رسم القيم المقدرة
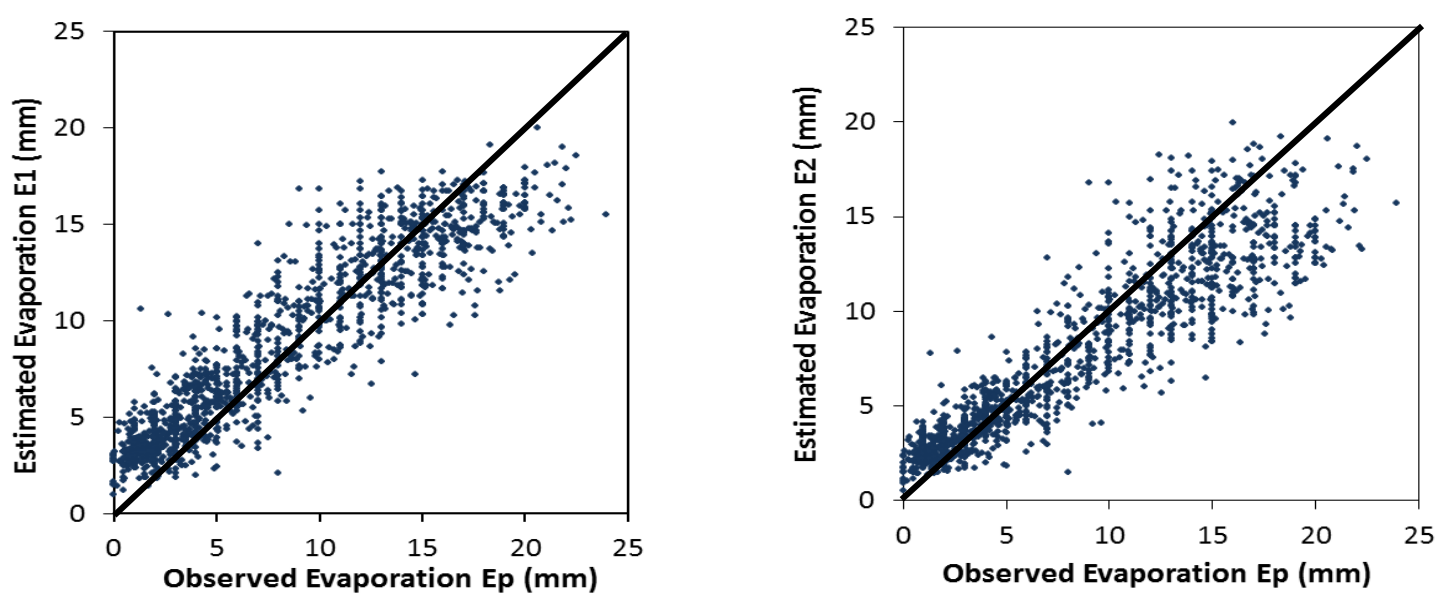

شكل (6) مخطط التبعثر للقيم المقدرة من نموذج (Linacre) اليومية 


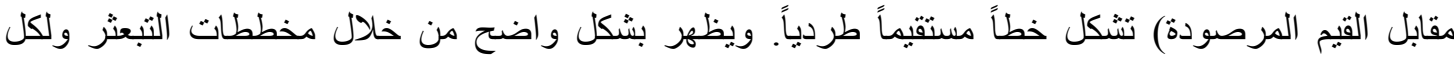

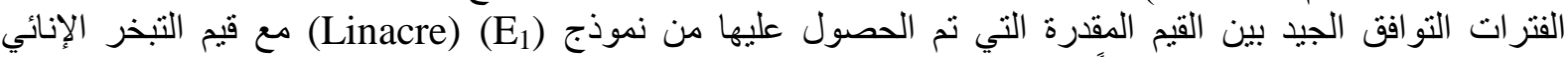

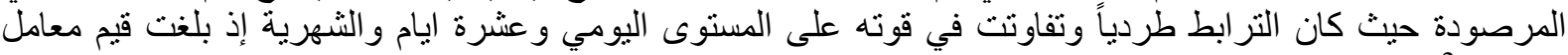
التحديد (R2) لها (87\%) و (93\%) و (95\%) على التى التو الي.
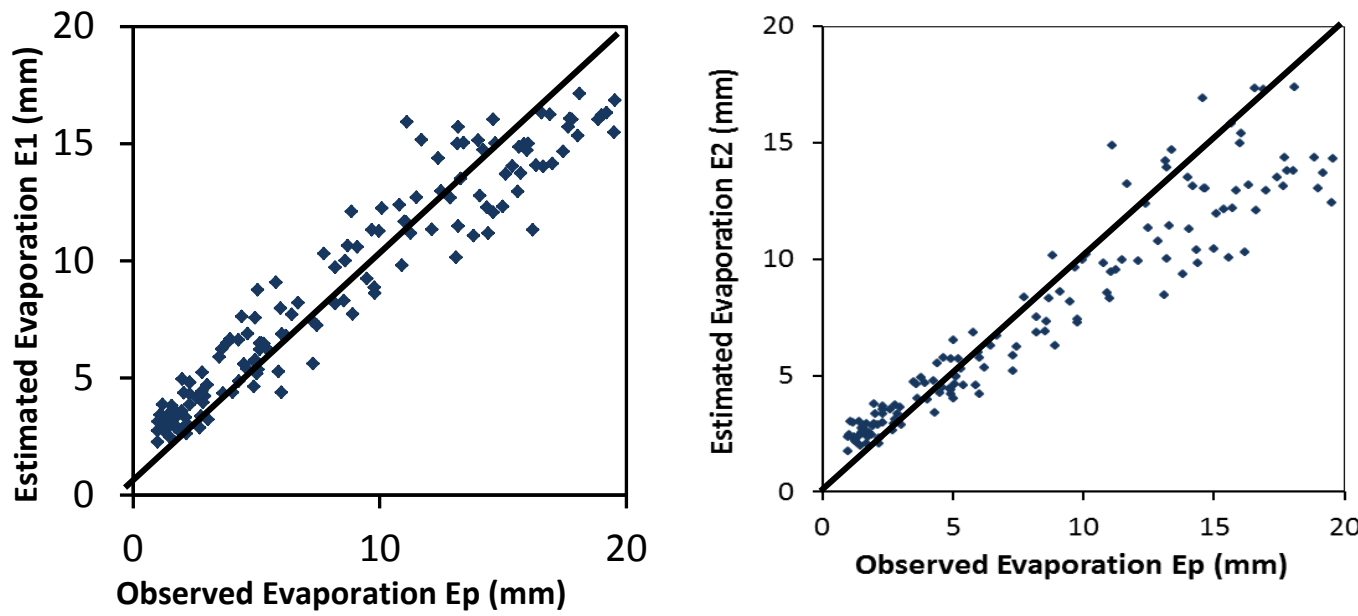

شكل (7) مخطط التبعثر للقيم المقدرة من نموذج (Linacre) لعشرة ايام
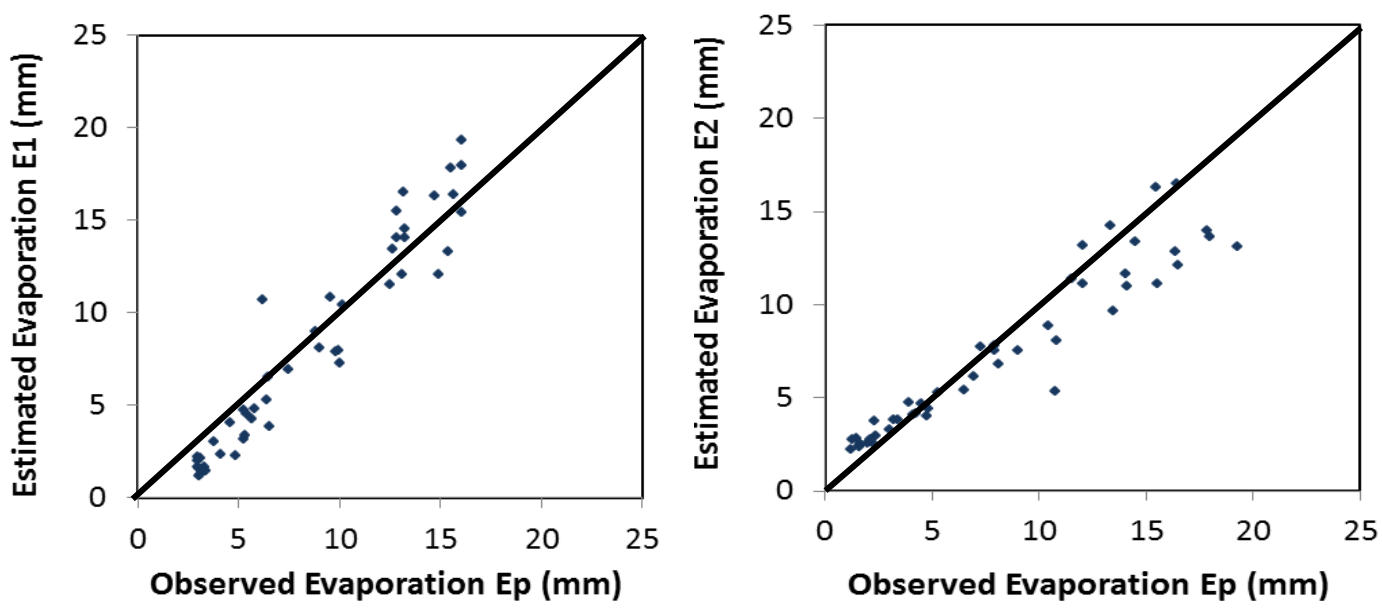

شكل (8) مخطط التبعثر للقيم المقدرة من نموذج (Linacre) الثهرية

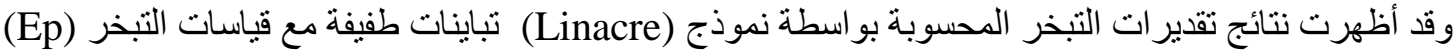

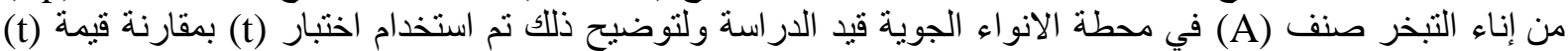

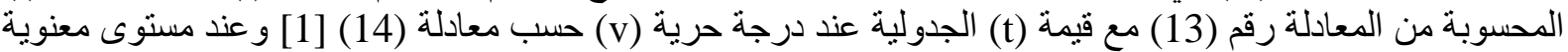

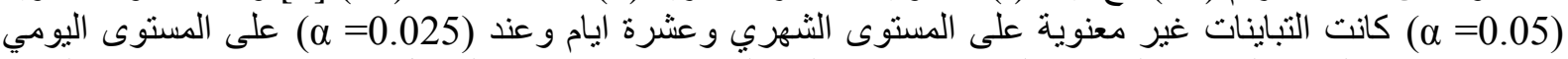
بحيث تفوّق قيم التبخر الثهرية المقدرة على مثيلاتها من القيم اليومية وعشرة ايام و الجدول (2) الاتي يين معدلات التئ التبخر $t=\frac{\left(\mu_{1}-\mu_{2}\right)}{\sqrt{\frac{\sigma_{1}^{2}}{n_{1}}+\frac{\sigma_{2}^{2}}{n_{2}}}}$ 


$$
v=\frac{\left(\frac{\sigma_{1}^{2}}{n_{1}}+\frac{\sigma_{2}^{2}}{n_{2}}\right)^{2}}{\frac{\left(\sigma_{1}^{2} / n_{1}\right)^{2}}{n_{1}-1}+\frac{\left(\sigma_{2}^{2} / n_{2}\right)^{2}}{n_{2}-1}}
$$

年: $\mu_{1}, \mu_{2}$ n n $_{1}, n_{2}$

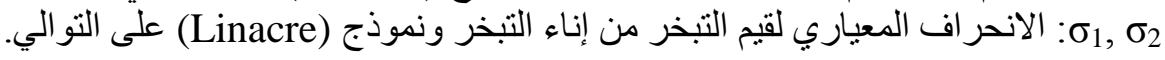

جدول (2) يمثل مقارنة بين التبخر الإنائي مع التبخر باستخدام معادلة (Linacre) على اساس شهري

\begin{tabular}{|c|c|c|c|}
\hline \multirow{2}{*}{ months } & \multicolumn{3}{|c|}{ Evaporation (mm/day) } \\
\cline { 2 - 4 } & $\mathbf{E}_{\mathbf{P}}$ & $\mathbf{E}_{\mathbf{1}}$ & $\mathbf{E}_{\mathbf{2}}$ \\
\hline Jan & 1.56 & 3.08 & 2.54 \\
\hline Feb & 2.06 & 3.27 & 2.64 \\
\hline Mar & 4.09 & 4.75 & 4.00 \\
\hline Apr & 5.88 & 6.53 & 5.31 \\
\hline May & 10.25 & 8.67 & 7.44 \\
\hline Jun & 15.15 & 13.13 & 12.06 \\
\hline Jul & 16.39 & 15.64 & 14.59 \\
\hline Aug & 15.79 & 15.43 & 13.87 \\
\hline Sep & 12.78 & 12.76 & 10.78 \\
\hline Oct & 7.814 & 9.68 & 7.48 \\
\hline Nov & 3.68 & 5.69 & 4.14 \\
\hline Dec & 1.76 & 3.64 & 2.84 \\
\hline $\begin{array}{c}\text { Mean } \\
\text { (mm/day })\end{array}$ & $\mathbf{8 . 1 0}$ & $\mathbf{8 . 5 2}$ & $\mathbf{7 . 3 1}$ \\
\hline $\begin{array}{c}\text { Sum } \\
\text { (mm/year) }\end{array}$ & $\mathbf{2 9 1 7}$ & $\mathbf{3 0 6 9}$ & $\mathbf{2 6 3 2}$ \\
\hline
\end{tabular}

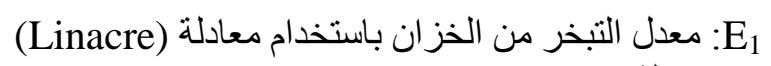

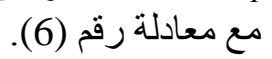

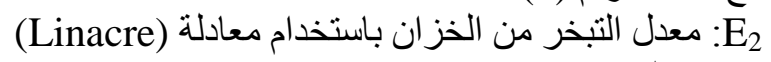

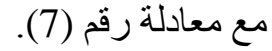
تم تقدير معدلات التبخر من خزان سد الموصل

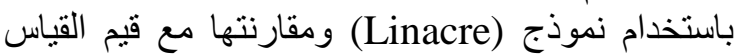

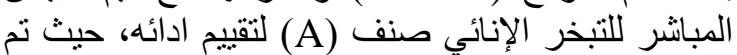
التوصل الى الاستنتاجات الاتية.

1. اظهرت النتائج من خلال مخططات التبعثر ولكل التبل الفترات التوافق الجيد بين القيم المقدرة التي تمعن

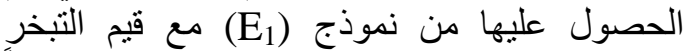

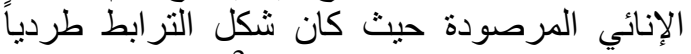

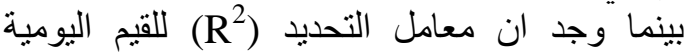

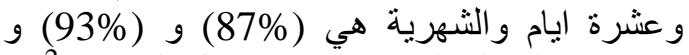

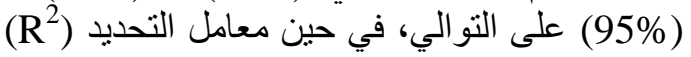

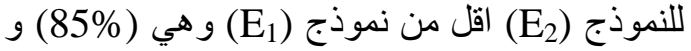

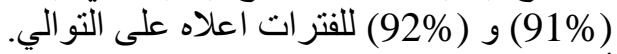

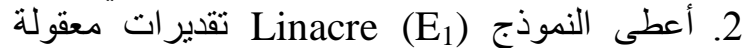

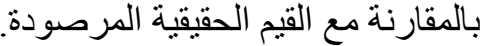

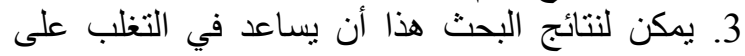

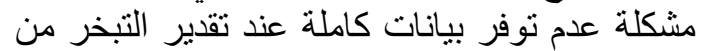
سطح المباه المفتوحة وتسهيل تطبيق النموذج.

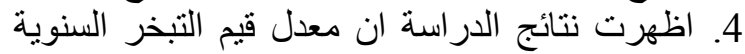

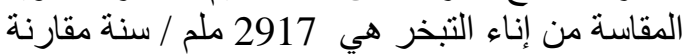
بنظيراتها من معدل القيم السنوية بكميات 3069

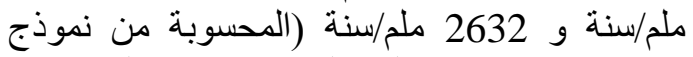

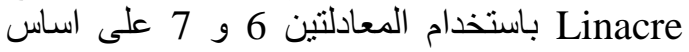
شهري) على التو الي.

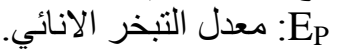

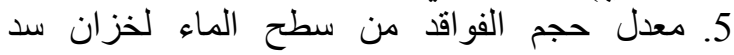
الموصل في حالة السعة التخزينية البالغة (11100)

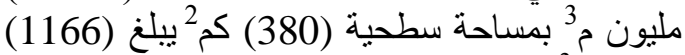

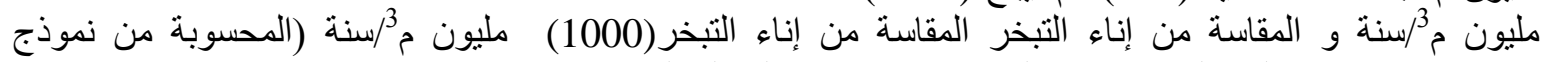
باستخدام المعادلتين 6 و 7 على اساس شهري) على التو التي. 
1. الراوي، خاثع محمود. (2000) "المدخل الى الاحصاء" الطبعة الثانية، كلية الزر اعة و الغابات، جامعة الموصل.

2. Allen, R.G., Pereira, L.S., Raes, D. and Smith M. (1998). "Crop Evapotranspiration: Guidelines for Computing Crop Water Requirements." Irrig. and Drain. Paper No. 56, FAO, Rome, Italy.

3. Allen. R., and Tasumi, M. (2005). "Evaporation from American Falls Reservoir in Idaho via a Combination of Bowen Ratio and Eddy Covariance" Proceedings 2005 EWRI Conf. Anchorage, Alaska.

4. Bruin, H. A. (1978). "A Simple Model for Shallow Lake Evaporation" Jour. Of Applied Meteorology, (78). 1132-1134.

5. Abdulla, T. M, and Hachum, A. Y. (1988). "Climatological Estimate of Saddam Reservoir Evaporation" Confidential Research, Mosul Dam Research Center.

6. Fadhil, A. I. (1990) " Optimal Operation of Reservoir on Tigris River " M.Sc. Thesis, Irrigation and Drainage Department, College of Engineering, University of Baghdad, Iraq.

7. Granger, R. J. and Hedstrom, N. (2010). "Modeling hourly rates of lake evaporation." Hydro. Earth Syst. Sci. Discuss., (7) 2727-2746. By: www. IVSL.org.

8. Hassani, A. Tajrishy, M. Abrishamchi, A. (2009). "Comparison of Several evaporation models applied to the Reservoir of Saveh Dam, Iran." International Perspective Environmental and Water Resources, Thailand.

9. Jensen, M.E., Burman, R.D. and Allen, R.G. (2010). "Estimating Evaporation From Water Surfaces." (ASCE) Manuals and Reports on Engineering Practice No 70, 2ed.

10. Keller, G. and Worrack, B. (1997)" Statistics for Management and Economics" Cole publishing Company, New York, p. 923.

11. Keskin, M. E., Terzi, O. and Taylan, D. (2004). "Fuzzy logic model approaches to daily pan evaporation estimation in western Turkey" Hydrological. Sciences. Journal, 49(6). P. 1001-1010. By: www. IVSL.org.

12. Lawrence, M. G. (2005). "The Relationship between Relative Humidity and the Dewpoint Temperature in Moist Air A Simple Conversion and Applications". American Meteorological Society, p. 225-233.

13. Linacre, E. T. (1977). "A simple formula for estimating evaporation rates in various climates, using temperature data alone." Agric. Forest Meteorol., 18, 409-424.

14. Nash, J. E. and Sutcliffe,J. V. (1970). "River flow forecasting through conceptual part 1- A discussion of principles" Journal of Hydrology, 10(3), 282-290. By: www. IVSL.org.

15. Piri, J. Amin, S. Moghaddamnia, A. Keshavarz, A. Han, D. and Remesan, R. (2009). "Daily Pan Evaporation Modeling in a Hot and Dry Climate." J. Hydro. Eng., (ASCE), 14(8), 803-811. By: www. IVSL.org.

16. Rayner, D. (2005). "Australian synthetic daily Class A pan evaporation." Technical Report, Department of Natural Resources and Mines, Queensland, Australia.

17. Senturk, K. and Oruk, F. (2010). "A Case Study: Evaporation Estimation at Oymapinar Dam" BALWOIS 2010 - Ohrid, Republic of Macedonia.

18. Tanny, J. Cohen, S. Assouline, S. Lange, F. Grava, A. Berger, D. Teltch, B. Parlange, M. (2008). "Evaporation from a small water reservoir: Direct measurements and estimates." J. Hydrol Eng. 351(1-2), 218-229. By: www. IVSL.org.

19. Vining, K. C. (2003). "Estimation of Monthly Evaporation from Lake Ashtabula in North Dakota, Orwell Lake in Minnesota, and Lake Traverse in Minnesota and South Dakota, 1931-2001." Water-Resources Investigations Report 03-4282.

$$
\text { تم اجراء البحث في كلية ألهنسة = جامعة ألموصل }
$$

